\title{
Ultra Wideband Bandpass Filter Using Microstrip-Slot Couplers Combined with Dumbbell Slots and H-Shaped Stubs
}

\author{
Amin Abbosh $^{1}$, Marek Bialkowski ${ }^{2}$, and David Thiel ${ }^{1}$ \\ ${ }^{1}$ Centre for Wireless Monitoring and Application, Griffith University \\ Nathan Campus, QLD4111, Australia \\ aabbosh@ieee.org \\ ${ }^{2}$ School of ITEE, The University of Queensland. \\ St Lucia, QLD4072, Australia
}

\begin{abstract}
A bandpass filter (BPF) that covers the ultra wideband (UWB) frequency range of $3.1 \mathrm{GHz}$ to $10.6 \mathrm{GHz}$ is presented. The filter uses multi-layer microstrip-slot couplers combined with dumbbell slots in the ground plane and $\mathbf{H}$-shaped stubs at the input/output ports to achieve high quality bandpass and band-rejection performance. The simulated results show that the designed filter has a UWB passband with an insertion loss less than $0.5 \mathrm{~dB}$ and a return loss greater than $25 \mathrm{~dB}$ at the centre of the passband. The device also exhibits sharp and wide low and high frequency stopbands. The simulated group delay of the filter indicates a low peak-to-peak variation of around 0.2ns across the passband. It features a compact size of $1.5 \mathrm{~cm} \times 2 \mathrm{~cm}$ when developed on RT6010 with dielectric constant of 10.2 .
\end{abstract}

Index Terms - Bandpass filter, multilayer, broadside coupling, slotted ground.

\section{INTRODUCTION}

Bandpass filters (BPF) are key devices in communication systems. For ultra wideband (UWB) applications, the BPF is required to have low insertion loss over the band $3.1 \mathrm{GHz}$ to $10.6 \mathrm{GHz}$, and a flat group delay performance within that band. Moreover, it should exhibit a very good selectivity below $3.1 \mathrm{GHz}$ and above $10.6 \mathrm{GHz}$ in order to meet the FCC spectrum mask.

A number of UWB BPF filters have already been reported in the microwave literature [1]-[12]. The early planar BPFs were designed using end-coupled coplanar waveguides (CPW) [1]. CPW BPFs based on the combination of lowpass and highpass periodic structures were also presented [2]. The parallel-coupled microstrip line with a slotted ground plane was employed to give a tight coupling for a wideband BPF [3]. In [4] a UWB filter was constructed by mounting a microstrip line in a lossy composite substrate so as to attenuate the signals at high frequencies In another method, a bandpass filter was designed by using two stopbands of a filter block with two tuning stubs on a ring [5]. In [6], a microstrip ring filter with dual stopbands, below $3.1 \mathrm{GHz}$ and above $10.6 \mathrm{GHz}$, was built to make up a UWB filter with sharp rejection. Compact UWB bandpass filters in microstrip technology were designed using multimode resonators [7][8].

In [9], a UWB BPF was developed by adopting a highpass filter prototype and transition stretch stubs to create the lower and upper stopbands. In [10], a technique for the design of ultra-wide bandpass filters with harmonic passband suppression is proposed. The design approach involves combining a standard UWB BPFs design with an electromagnetic bandgap periodic structure.

In [11], a BPF is composed of five short-circuited stubs separated by connecting lines that contribute to the filter's selectivity. In addition, a cross-coupling between the input/output feed lines is introduced to generate new pairs of attenuation poles at each side of the passband. Although performance of the filter shows a sharp cutoff at 3 and 12 $\mathrm{GHz}$, it has a narrow low and high stopbands. Moreover, the manufacturing process is complicated as it needs many vias.

The tolerance of the microstrip and CPW fabrication process imposes an upper limit upon coupling levels for parallel- and edge-coupled structures. This makes the manufacturing of UWB filters which utilize those structures difficult as their performance is very sensitive to manufacturing errors. This difficulty can be circumvented by implementing tight coupling using broadside coupling technique [12]. In [12], elliptical shaped broadside microstrip-slot couplers were used to construct UWB bandpass filters. In order to improve the performance at the high stopband, multiple broadside-coupled sections of up to five were utilized. The drawback of this approach is an increased size and degradation of passband characteristics.

In this paper, the shortfalls of the design presented in [12] are overcome by combining two multilayer microstrip-slot couplers with dumbbell slots in the ground plane and $\mathrm{H}$ shaped open-ended shunt stubs at the input and output ports. This approach reduces the overall size of filter and enhances the high stopband performance. The advantages of the new design are demonstrated via full-wave electromagnetic simulations.

\section{PRoposed DEVICE}

The proposed BPF configuration, whose middle part is based on an initial broadside-coupled multilayer configuration of [12], is shown in Fig.1. The structure includes three conductive layers interleaved with two substrates. The top layer (Fig. 1a) and the bottom layer (Fig.1c) have two similar elliptical shaped microstrip patches which are coupled via an elliptical slot at the mid layer of the structure (Fig.1b). 


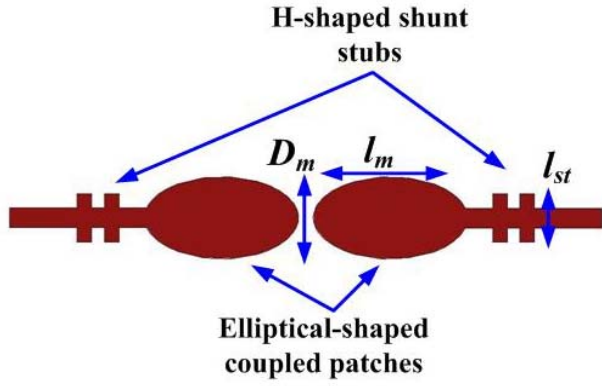

(a) Top layer

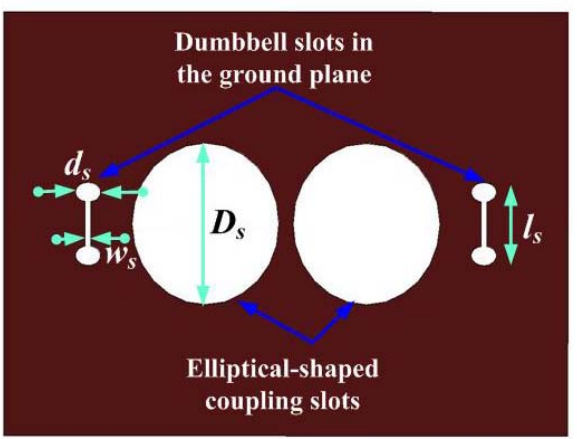

(b) Mid layer

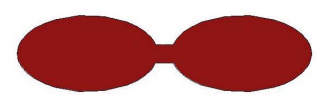

(c) Bottom layer

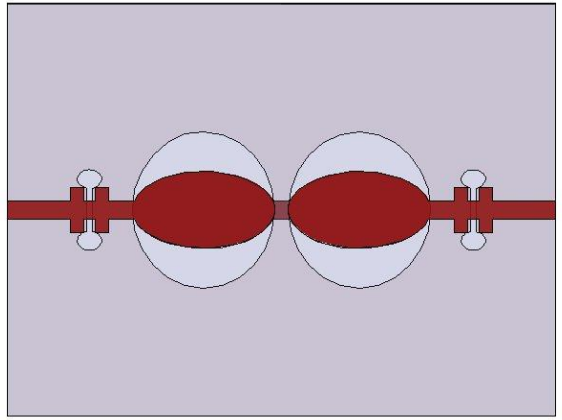

(d) Top view of the whole structure

Top layer

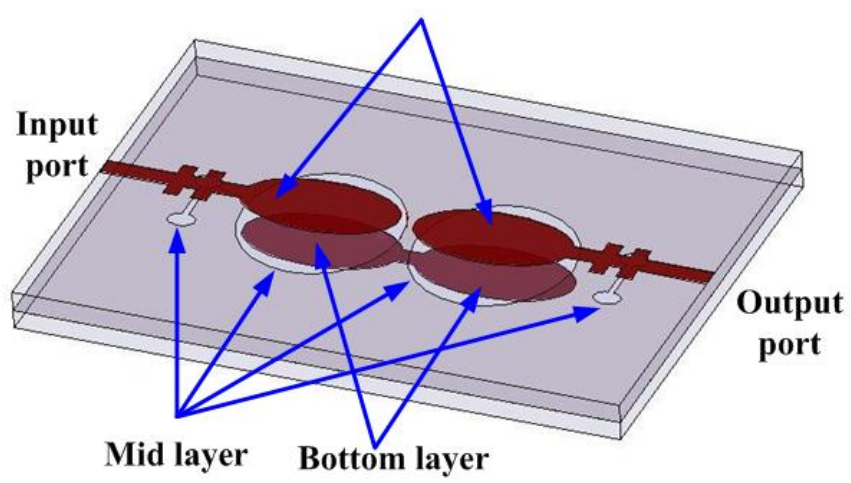

(e) Side view of the whole structure

Fig.1.Configuration of the proposed multilayer broadsidecoupled bandpass filter.
The ground plane of the whole structure is located in the mid layer. The reason for the choice of this broadsidecoupled configuration is that it provides an almost constant tight coupling, which is important to achieve the required filter's characteristics, across the ultra wideband as indicated in [13]-[14].

The main modification introduced in this paper is the inclusion of a coupled dumbbell slot in the ground plane (mid layer) as shown in Fig. $1 \mathrm{~b}$ and $\mathrm{H}$-shaped shunt open-ended stubs connected with the input and output ports at the top layer as revealed in Fig. 1a. This added circuit behaves as a low pass filter, which improves the high stopband characteristics of the entire filter without the need to add more middle sections.

The design procedure for the proposed filter includes two main steps: The first step concerns designing the two-section broadside-coupled structure to accomplish a passband that extends from 3.1 to $10.6 \mathrm{GHz}$, whereas the second step is to design the combined slotted ground plane and open-ended shunt stubs to have a sharp cutoff at frequency larger than $10.6 \mathrm{GHz}$.

Concerning the broadside-coupled part of the filter, the detailed design method presented in [12]-[14] is applied to find its dimensions assuming that the passband of the filter covers the ultra wideband 3.1 to $10.6 \mathrm{GHz}$. It is clear from the theoretical analysis of [12] that the important parameter that defines the design procedure is the required value for the coupling factor $C$. Following the analysis presented in [12], it is possible to show that the effective scattering parameters for the two-section broadside-coupled structure ( $S_{11 e f}$ and $S_{21 e f}$ ) are given as [12];

$$
\begin{gathered}
S_{11 e f}=S_{11}+\frac{S_{21}^{2} S_{11}}{1-S_{11}^{2}} \\
S_{21 e f}=\frac{S_{21}^{2}}{1-S_{11}^{2}} \\
S_{11}=\frac{1-C^{2}\left(1+\sin ^{2}\left(\beta_{e f} l\right)\right)}{\left[\sqrt{1-C^{2}} \cos \left(\beta_{e f} l\right)+j \sin \left(\beta_{e f} l\right)\right]^{2}} \\
S_{21}=\frac{j 2 C \sqrt{1-C^{2}} \sin \left(\beta_{e f} l\right)}{\left[\sqrt{1-C^{2}} \cos \left(\beta_{e f} l\right)+j \sin \left(\beta_{e f} l\right)\right]^{2}}
\end{gathered}
$$

where $\beta_{e f}$ is the effective phase constant in the medium of the coupled structure, and $l$ is the physical length of the coupled structure which is chosen such that; $\beta_{e f} l=\pi / 2$ at the centre of the passband $(6.85 \mathrm{GHz})$.

For the configuration under investigation, it is possible to show that [12];

$$
\beta_{e f}=\frac{\beta_{e}+\beta_{o}}{2}=2 \pi \sqrt{\varepsilon_{r}} / \lambda
$$


where $\beta_{e}$ and $\beta_{o}$ are the phase constants for the even- and odd-mode respectively, $\lambda$ is the free space wavelength, and $\varepsilon_{r}$ is the dielectric constant of the substrate.

Solving (1)-(5) for the coupling factor $C$ that gives the best possible performance across the passband (3.1 to 10.6 $\mathrm{GHz}$ ) results in $C=0.7$. Using this value with the design approach for the broadside-coupled structures [13] results in the following values for the design parameters shown in Fig. 1 assuming that the substrate is Rogers RT6010 (with $\varepsilon_{\boldsymbol{r}}=10.2$, thickness $=0.635 \mathrm{~mm}$, and tangent loss $\left.=0.0023\right)$; $D_{m}=2.8 \mathrm{~mm}, D_{s}=5.7 \mathrm{~mm}, l_{m}=5.2 \mathrm{~mm}$.

The broadside-coupled structure designed in the previous steps results in a BPF that has the required passband (3.1 to $10.6 \mathrm{GHz}$ ) and good low frequency stopband (below 3.1 $\mathrm{GHz}$ ). However, the performance at the high stopband shows a slow cutoff and a spurious response that appears at integer multiple of the midband frequency [12]. The most pronounced spurious response is the one that appears at 13.7GHz.

To improve the performance of the BPF at the high stopband, H-shaped shunt open-ended stubs connected with the microstrip line of the top layer and coupled with dumbbell shaped slots at the ground plane of the mid layer are employed. The slotted ground plane disturbs the current distribution in the ground plane, and thus changes the line capacitance and inductance of a transmission, whereas the shunt stubs added a reactive element to the transmission line. The equivalent circuit for the combination of the slotted ground plane and shunt open-ended stubs is as shown in Fig. 2 after using the approach presented in [15], [16]. This circuit forms a LPF with a wide high stopband.

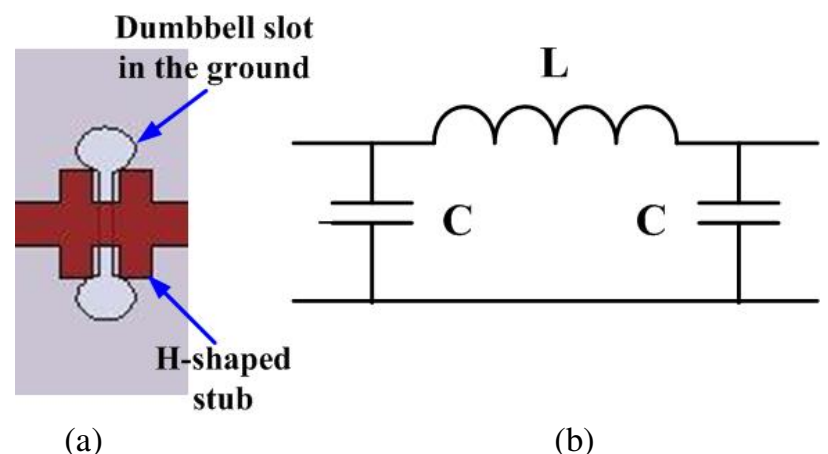

Fig.2 Configuration of the LPF (a) and its equivalent circuit (b).

The rough design for the LPF shown in Fig. 2 is to choose length of the stubs and the slot in the ground plane to have half of the effective wavelength at the required cutoff frequency. For the proposed filter, the cutoff frequency is chosen to be at the first spurious response of the broadsidecoupled structure, i.e. at $13.7 \mathrm{GHz}$. After optimization using the software CST Microwave Studio, length of the slot in the ground plane $\left(l_{s}\right)$ and of the shunt stubs at the top layer $\left(l_{s t}\right)$ are equal to $2 \mathrm{~mm}$ and $1.7 \mathrm{~mm}$, respectively, whereas width of the slot in the ground plane was fixed at $0.2 \mathrm{~mm}$, width of the shunt stubs is $0.5 \mathrm{~mm}$, and radius of the circular slots that form the two ends of the dumbbell slot is $0.4 \mathrm{~mm}$. In finding the above mentioned values, the substrate was considered to be Rogers RT6010 with thickness of each layer equal to $0.635 \mathrm{~mm}$.

\section{RESULTS \& DISCUSSIONS}

In order to verify performance of the proposed BPF, the device is simulated using the software CST Microwave Studio. The overall dimension of the designed filter used in the simulations including the microstrip feeders at the input and output is $1.5 \mathrm{~cm} \times 2 \mathrm{~cm}$ indicating a compact device.

Variation of the scattering parameters $\left(\mathrm{S}_{11} \& \mathrm{~S}_{21}\right)$ with frequency is shown in Fig. 3.

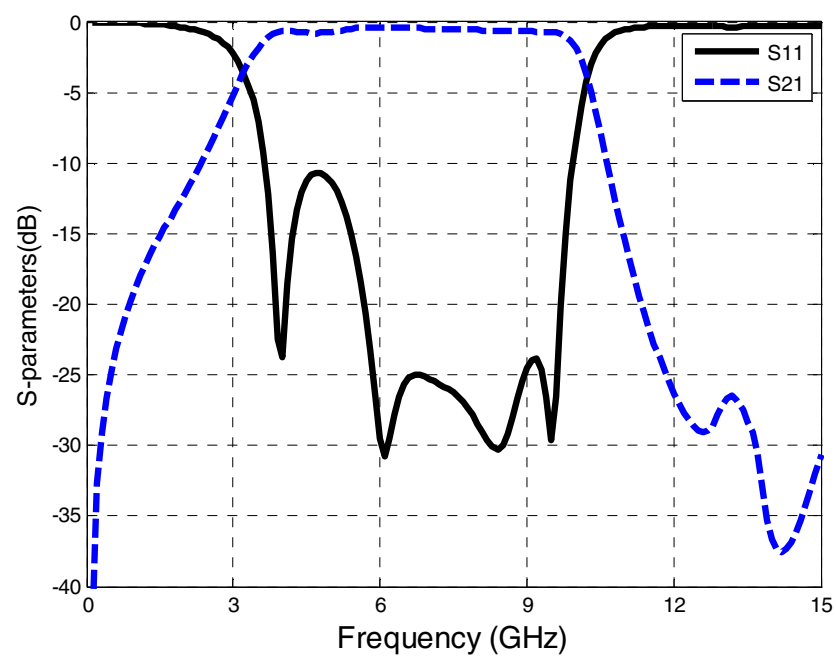

Fig.3 Variation of the scattering parameters $\left(\mathrm{S}_{11} \& \mathrm{~S}_{21}\right)$ with frequency.

It is apparent from Fig. 3 that the designed filter has a passband which covers the ultra wideband range of 3.1 to 10.6GHz. The insertion loss at the centre of the passband is less than $0.5 \mathrm{~dB}$, whereas the return loss is larger than $25 \mathrm{~dB}$. Also, the results of Fig. 3 reveal the sharp low and high frequency stopbands. The sharp low frequency stopband is a natural behavior of the broadside-coupled structure used in the configuration [12], whereas the sharp stopband at the high frequency is due to the used lowpass filter which consists of the dumbbell slot in the ground plane and H-shaped shunt open-ended stubs connected with the input/ output ports at the top layer.

Effect of incorporating the coupled H-shaped stubs and dumbbell shaped slot on the performance of the BPF can be clarified if the performance of the two-section broadsidecoupled BPF designed in this paper is compared with performance of the two-section broadside-coupled BPF that does not include the coupled H-shaped shunt stub/ dumbbell slot as presented in [12]. It is obvious from the comparison that although the two structures have the same size and the same passband and low stopband performance, the modified structure of the BPF presented in this paper has a sharper and wider high stopband. Also, the design presented in [12] has a spurious response at the high stopband. In [12], five sections 
of broadside-coupled structures were utilized to improve the performance of the BPF at the high stopband.

For impulse radio systems, the BPF is strongly required to have a flat group delay across the passband to keep the distortion of the pulse shape to minimum. Thus, variation of the group delay of the designed filter should have a subnanosecond peak-to-peak variation across the passband. The simulated results of the group delay for the proposed filter is shown in Fig. 4 which depicted a peak-to-peak variation in the group delay of $0.2 \mathrm{~ns}$ across the band $3.5 \mathrm{GHz}$ to $9.5 \mathrm{GHz}$.

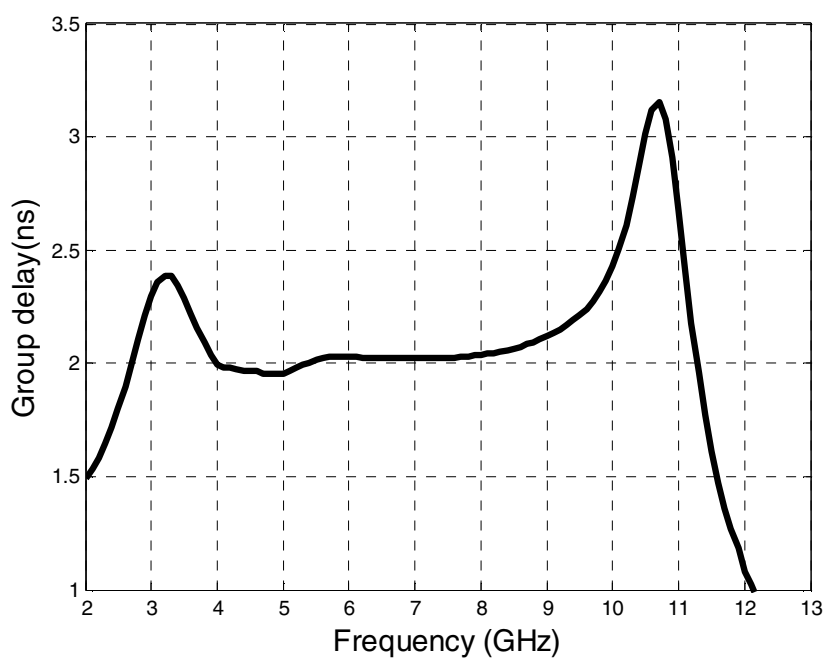

Fig.4 Variation of the group delay with frequency.

\section{CONCLUSION}

A bandpass filter that covers an ultra wideband frequency from 3.1 to $10.6 \mathrm{GHz}$ has been presented. The device uses microstrip-slot couplers to achieve bandpass operation. To improve the cutoff performance at the high frequency band and to extend the high stopband, a combination of dumbbell slot in the ground plane and H-shaped open-ended stubs connected to the input/output ports is utilized. The simulated results have shown that the designed filter has a passband that extends from $3.1 \mathrm{GHz}$ to $10.6 \mathrm{GHz}$ with an insertion loss which is less than $0.5 \mathrm{~dB}$ and a return loss which is larger than $25 \mathrm{~dB}$ at the centre of the passband. The results have also shown sharp low and high frequency stopbands. The high stopband is wide as it extends to above $15 \mathrm{GHz}$. The simulated group delay of the filter has indicated a peak-topeak variation of around $0.2 \mathrm{~ns}$. This reveals the distortionless performance of the filter when used with ultra wideband pulsed systems. The device has a compact size of $1.5 \mathrm{~cm} \times 2 \mathrm{~cm}$, which is welcome in many applications. At the time of writing this paper, the filter is in the manufacturing stage.

\section{REFERENCES}

[1] F. Lin, C. Chiu, and R. Wu, "Coplanar waveguide bandpass filter-A ribbon-of-brick-wall design," IEEE Trans. Microwave Theory Tech., vol.43, pp.1589-1596, 1995.

[2] H. Hu, X. Huang, and C. Cheng, "Ultra-wideband bandpass filter using CPW-to-microstrip coupling structure," Electronics Letters, vol.42, no.10, pp., 2006.

[3] M. Mandal ,and S. Sanyal, "Compact wideband bandpass filter," IEEE Microw. Wireless Compon. Lett., vol. 16, no. 1,pp.46-48, 2006.

[4] A. Saito, H. Harada, and A. Nishikata, "Development of band pass filter for ultra wideband (UWB) communication," Proc. IEEE Conf. Ultra Wideband Systems Technology, pp. 76-80, 2003.

[5] L. Hsieh, and K. Chang, "Compact, low insertion-loss, sharp-rejection, and wide-band microstrip bandpass filters," IEEE Trans. Microwave Theory Tech., vol. 51, no. 4, pp. 1241-1246, 2003.

[6] H. Ishida, and K. Araki, "Design and analysis of UWB bandpass filter with ring filter," in IEEE MTT-S Int. Dig., vol. 3, pp. 1307-1310, 2004.

[7] L. Zhu, S. Sun, and W. Menzel, "Ultra-wideband (UWB) bandpass filters using multiple-mode resonator," IEEE Microw. Wireless Compon. Lett., vol. 15, no. 11, pp. 796-798, 2005.

[8] J. Gao, L. Zhu, W. Menzel, and F. Bögelsack, "Shortcircuited CPW multiplemode resonator for ultrawideband (UWB) bandpass filter," IEEE Microwave Wireless Compon. Lett., vol.16, no.3, 104-106, 2006.

[9] T. Kuo, S. Lin, and C. Chen, "Compact ultra wideband bandpass filters using composite microstrip-coplanarwaveguide structure," IEEE Trans. Microwave Theory Tech., vol. 54, no. 10, pp. 3772-3778, 2006.

[10] J. García-García, J. Bonache, and F. Martín, "Application of electromagnetic bandgaps to the design of ultra-wide bandpass flters with good out-of-band performance," IEEE Trans. Microwave Theory Tech., vol. 54, no. 12, pp. 4136-4140, 2006.

[11] H. Shaman, and J. Hong, "A novel ultra-wideband (UWB) bandpass filter (BPF) with pairs of transmission zeroes," IEEE Microw. Wireless Compon. Lett., vol. 17, no.2, pp.121-123, 2007.

[12] A. Abbosh, "Planar bandpass filters for ultra wideband applications," IEEE Trans. Microwave Theory Tech., vol. 55, no. 10, pp. 2262-2269, 2007.

[13] A. Abbosh, and M. Bialkowski, "Design of compact directional couplers for UWB applications," IEEE Trans. Microwave Theory Tech., vol. 55, no.2, pp. 189194, 2007.

[14] A. Abbosh, "Ultra wideband phase shifters," IEEE Trans Microwave Theory Tech, vol.55, no.9, pp.1935-1941, 2007.

[15] J. Lim, C. Kim, D. Ahn, Y. Jeong, and S. Nam, "Design of low-pass filters using defected ground structure," IEEE Trans. Microw. Theory Tech., vol. 53, no. 8, pp. 2539-2545, 2005.

[16] N. Karmakar, S. Roy, I. Balbin, "Quasi-static modeling of defected ground structure," IEEE Trans. Microw. Theory Tech., vol. 54, no. 5, pp. 2160-2168, 2006. 